\title{
Statistical characterization of gas-patch distributions in partially saturated rocks
}

\author{
Julianna Toms-Stewart ${ }^{1}$, Tobias M. Müller ${ }^{2}$, Boris Gurevich ${ }^{3}$, and Lincoln Paterson ${ }^{4}$
}

\begin{abstract}
Reservoir rocks are often saturated by two or more fluid phases forming complex patterns on all length scales. The objective of this work is to quantify the geometry of fluid phase distribution in partially saturated porous rocks using statistical methods and to model the associated acoustic signatures. Based on X-ray tomographic images at submillimeter resolution obtained during a gasinjection experiment, the spatial distribution of the gas phase in initially water-saturated limestone samples are constructed. Maps of the continuous variation of the percentage of gas saturation are computed and associated binary maps obtained through a global thresholding technique. The autocorrelation function is derived via the two-point probability function computed from the binary gas-distribution maps using Monte Carlo simulations.
\end{abstract}

The autocorrelation function can be approximated well by a single Debye correlation function or a superposition of two such functions. The characteristic length scales and show sensitivity (and hence significance) with respect to the percentage of gas saturation. An almost linear decrease of the Debye correlation length occurs with increasing gas saturation. It is concluded that correlation function and correlation length provide useful statistical information to quantify fluid-saturation patterns and changes in these patterns at the mesoscale. These spatial statistical measures are linked to a model that predicts compressional wave attenuation and dispersion from local, wave-induced fluid flow in randomly heterogeneous poroelastic solids. In particular, for a limestone sample, with flow permeability of 5 darcies and an average gas saturation of $\sim 5 \%$, significant $\mathrm{P}$-wave attenuation is predicted at ultrasonic frequencies.

\section{INTRODUCTION}

Understanding how the percentage of two (or more) pore fluids affects $P$-wave velocities is important for interpreting time-lapse seismic data, particularly for tracking fluid-front movements. Application of Gassmann's fluid-substitution relations (Gassmann, 1951), in addition to fluid-mixing equations such as those of Brie et al. (1995) or Wood (1941), is one approach that could make forward-modeling estimates of P-wave velocities at different percentages of fluid saturation. However, the problem with these approaches is that partial fluid-saturation effects such as mesoscopic fluid flow are ignored completely.

Mesoscale fluid flow arises when a passing wave induces different fluid pressures in regions of rock saturated by different fluid types, where mesoscale specifically refers to a length scale greater than pore scale but less than wavelength scale. The presence of spatial gradients in fluid pressure causes fluid to flow relative to the rock frame. This dissipates energy and results in the attenuation and dispersion of a propagating waveform. A number of different patchy saturation models accounting for the mesoscale distribution of fluid heterogeneities have been proposed: the concentric sphere model of White et al. (1976), the acoustics of patchy saturation (APS) model of Johnson (2001), and the continuous-random media (CRM) model of Toms et al. (2006, 2007). These theoretical models allow us to calculate dynamic-equivalent elastic moduli as a function of percentage of fluid saturation and wave frequency. However, one must assume either a fixed geometry of the patch distribution (such as periodically distributed spherical inclusions in White's model) or fluid distribution given by a specific correlation function (for CRM). Thus, the applicability of these models hinges on knowledge of the

Manuscript received by the Editor 31 January 2008; revised manuscript received 23 May 2008; published online 11 March 2009.

${ }^{1}$ Formerly Curtin University of Technology, Perth, Australia; presently ExxonMobil, Upstream Research Company, Houston, Texas, U.S.A. E-mail: julianna .toms@exxonmobil.com.

${ }_{3}^{2}$ Formerly University of Karlsruhe, Karlsruhe, Germany; presently CSIRO Petroleum, Perth, Australia. E-mail: tobias.mueller@ csiro.au.

${ }^{3}$ Curtin University of Technology \& CSIRO Petroleum, Perth, Australia. E-mail: b.gurevich@ @urtin.edu.au.

${ }^{4}$ CSIRO Petroleum, Melbourne, Australia. E-mail: lincoln.paterson@csiro.au.

(C) 2009 Society of Exploration Geophysicists. All rights reserved. 
real spatial distribution of pore fluids in rocks. The spatial distribution is influenced by rock heterogeneity, the prior history of fluid movement, and density and viscous effects such as viscous fingering (Homsy, 1987).

The most suitable tool to assist with imaging fluid distributions of partially saturated rocks is X-ray tomography. It is already routinely applied in petroleum engineering to image reservoir lithologies undergoing secondary and tertiary recovery processes (Wellington and Vineagar, 1987; Dunsmuir, 1991; Withjack et al., 2003). X-ray tomography increasingly is used to characterize soils (Peyton et al., 1992; De Gryze et al., 2006) and rocks (Arns et al., 2002), to produce input models for numerical algorithms that calculate transport properties of rocks (Arns et al., 2001; Arns et al., 2004; Arns et al., 2005), and to calculate elastic properties (Arns et al., 2002). In fact, it is being used in many applications spanning the geoscience field (Ketcham and Carlson, 2001).

The simultaneous acquisition of X-ray tomography images and acoustic measurements during fluid-saturation experiments on porous rock will provide the most direct means of relating velocity-saturation information to pore-fluid distribution. There have been several studies of this kind (Cadoret et al., 1995; Cadoret et al., 1998; Monsen and Johnstad, 2005). Cadoret et al. (1995) use X-ray tomography images to explain why velocities at the same percentage of water saturation may differ for drainage and imbibition experiments. Where the velocities differ, the drainage images show the presence of distinct gas-bearing clusters (for water saturations $>80 \%$ ), but the imbibition images show no such clustering. This suggests that differences in fluid distribution will cause differences in velocity measurements. The same conclusion has been drawn for attenuation measurements (Cadoret et al., 1998).

Although X-ray tomography images are central to the experiments of Cadoret et al. (1995) and Monsen and Johnstad (2005), the images themselves are analyzed only qualitatively. Apart from identifying images that have clustering (Cadoret et al. 1995) or show fluid displacement in preferred directions (Monsen and Johnstad, 2005), no further analysis of fluid-saturation patterns is performed. Thus, variations in velocities and attenuation are linked qualitatively to changes in fluid-saturation patterns; missing is a quantitative description.

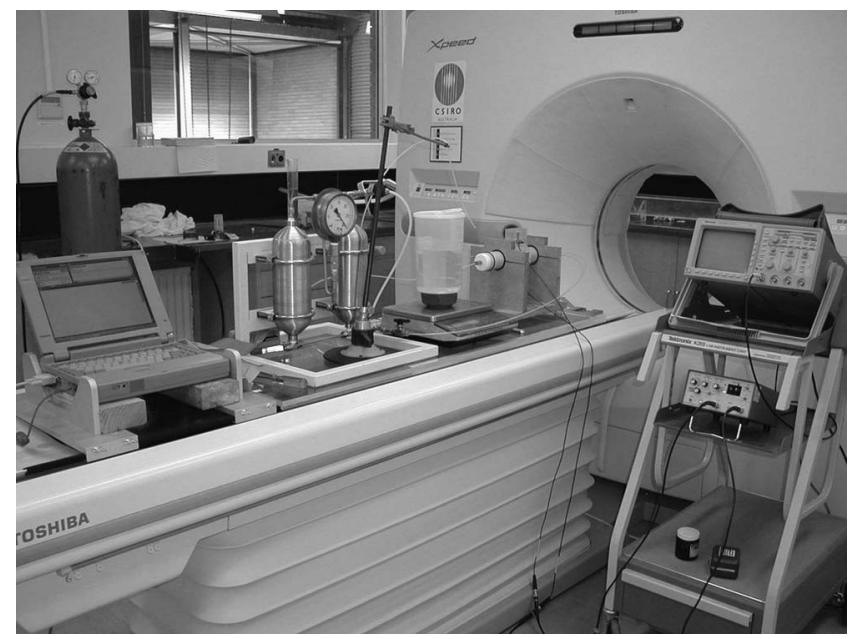

Figure 1. Experimental setup for imaging the core sample during the fluid-replacement experiment.
We want to improve understanding of the geometric characteristics of real immiscible fluid distributions in rock and to model their associated acoustic signatures. To do so, we examine a series of X-ray tomography images (with mesoscale resolution) obtained from a drainage experiment performed on Mount Gambier Limestone samples (Lincoln Paterson and Gregory Lupton, personal communication, 2003). The objective is to analyze fluid-saturation patterns quantitatively using statistical methods, thus showing how they can be described and how they vary as saturation changes. In particular, the applicability and significance of correlation measures on mesoscopic fluid-patch patterns is investigated. Moreover, we want to develop a modeling strategy by combining the statistical measures inferred from the X-ray tomography images and the theory of statistical wave propagation applied to the patchy saturation problem (Toms et al., 2007). This approach allows us, in principle, to obtain reliable estimates of attenuation and dispersion.

The paper is organized as follows. We briefly describe a recent X-ray tomography experiment of partially saturated limestone samples. Then we explain the construction of gas-saturation maps and their associated binary maps based on X-ray tomography results. This is followed by a statistical analysis of the binary maps. We introduce the concept of indicator and correlation functions for mesoscopic heterogeneity. The spatial correlations as a function of saturation and rock type are analyzed in detail. Two characteristic length scales, the Debye correlation length (Debye and Bueche, 1949) and the mean length, are evaluated for the gas-saturation maps. Then we show how these statistical measures can be incorporated into a model of acoustic signatures of partially saturated rocks. We evaluate estimates of P-wave attenuation and velocity dispersion to be expected in these partially saturated rocks. Finally, we present conclusions and recommendations for future research.

\section{GENERATING GAS-SATURATION MAPS USING XRT}

\section{Experimental setup}

Paterson and Lupton (personal communication, 2003) have performed a series of drainage experiments on Mount Gambier Limestone samples. The experimental setup is shown in Figure 1. A cylindrical core sample encased in polyvinyl chloride (PVC) pipe is connected to piping that controls pressured fluid injection of nitrogen gas and subsequent water extraction. The medical X-ray imager is shown in the background; at various stages during the saturation experiment, images are taken of the core sample.

The experiment proceeds initially by taking an image of the dry core sample. The core sample is then fully saturated with water. A vacuum is applied to ensure uniform saturation of the water throughout the pore space and to draw out remaining air. An image is then taken of the fully water-saturated sample. Fluid replacement begins by injecting pressurized nitrogen gas into the end of the core. The flow rate is kept at a constant pressure (but not at a constant rate) throughout the experiment. Images are taken at different times.

The core samples are Mount Gambier Limestone (MGL) from the Bruhn quarry in South Australia (Bruhn Building Stone, 2008). They generally have a high permeability (around 5 darcies) and typical effective porosities in the range of $36 \%-44 \%$. Although the saturation experiments were performed on a number of different core samples, only one experiment (performed on sample MGL 2a) is suitable for thorough analysis, as images are taken at both low and high gas saturations. 
Imaging of pore spaces and individual mineral grains requires the use of high-resolution tomography machines. Typically, pore-scale imaging of most materials requires resolutions of 3-5 $\mu \mathrm{m}$ (Olafuyi et al., 2006). When combined with a typical field of view of 512 $\times 512$ or $1024 \times 1024$ pixels, this limits the size of the scanned sample to $5 \mathrm{~mm}-1 \mathrm{~cm}$. In Figure 2, we show a pore-scale image of a typical sample of MGL containing no saturating fluids. The MGL samples have a very complicated microstructure composed of macroporosity and microporosity (not resolvable at this scale). Variations in rock porosity have a significant effect on the distribution of different pore fluids.

Pore-scale images of a drainage experiment performed on a Berea sandstone and monodisperse bead pack show that the wetting fluid is present as pendular rings, bridges between adjacent grains, and lenses in pore throats (Turner et al., 2004). This degree of detail is not visible in the images obtained during the Paterson and Lupton experiments because the resolution of the medical imager utilized was poorer but the field of view was larger. The relatively large core samples have a radius of $0.050 \mathrm{~m}$ and a length of $0.24 \mathrm{~m}$ and were imaged with a minimum pixel size of $0.36 \mathrm{~mm}$. Hence, to visualize changes in fluid distribution, the images must be processed as specified in the following section.

\section{Basic X-ray computed-tomography theory}

The X-ray computed-tomography (CT) method was developed originally for medical applications - specifically, the imaging of bones and soft tissue (Hounsfield, 1972). Typical medical X-ray tomography machines, such as the one shown in Figure 1, use a single source rotated around the scanned object. The source is an X-ray tube that emits a beam of photons received by a fixed set of detectors ringed around the object. The detectors measure the intensity of the received X-ray, which is related to the intensity of the source $\mathrm{X}$-ray for homogeneous materials by Beer's law (Wellington and Vinegar, 1987):

$$
I=I_{0} \exp (-\mu x)
$$

where $\mu$ is the linear-attenuation coefficient of the scanned material and $x$ is the distance traveled through the material. When the material is heterogeneous, Beer's law is

$$
I=I_{0} \exp \left(-\sum_{i} \mu_{i} x_{i}\right),
$$

where $\mu_{i}$ is the linear-attenuation coefficient of each material and $x_{i}$ is the distance traveled through each material $i$.

Different types of heterogeneities (materials) in a porous rock, such as saturating fluids and mineral grains, can be imaged, provided there is sufficient contrast in the linear X-ray attenuation coefficient of each type of material. The linear-attenuation coefficient, in turn, depends on how atoms in a material absorb and scatter energy (see Wellington and Vineagar, 1987).

Although the detectors measure the intensity of the received X-rays, the output of a CT scanner is in Hounsfield units (HU). Hounsfield units come from the reconstruction of linear-attenuation coefficients from the measured X-ray intensities using methods such as the Shepp-Logan filter (Shepp and Logan, 1974). In general, medical imagers are calibrated such that the CT value of water is zero $\mathrm{HU}$ and the CT value of air is $-1000 \mathrm{HU}$. However, other types of calibration (Johns et al., 1993; Orsi and Anderson, 1999) can be adopted that relate CT values linearly to a materials bulk density; this is especially useful for geologic applications.

Our approach relates CT values to the percentage of gas saturation by assuming that the CT value can be related linearly to the CT values of water, gas, and mineral grains in proportion to their respective volume fraction. This approach is outlined in the next section.

\section{Processing X-ray CT images}

As the resolution of our medical imager is not sufficient to image the interface between gas, water, and mineral grains, the CT value of each pixel is assumed approximately equal to the average of CT responses because of the different percentages of mineral grains, pore spaces, and pore fluids. Thus, dry-map pixels have CT values (see Figure 3a) given by

$$
\mathrm{CT}_{\mathrm{dry}} \approx(1-\phi) \mathrm{CT}_{\text {grain }}+\phi \mathrm{CT}_{\mathrm{air}},
$$

where $\phi$ is rock porosity, and $\mathrm{CT}_{\text {grain }}$ and $\mathrm{CT}_{\text {air }}$ are the $\mathrm{CT}$ values of the grains and air-filled pore space, respectively. Pixels belonging to the fully water-saturated map have CT values (see Figure 3b) given by

$$
\mathrm{CT}_{\text {full sat }} \approx(1-\phi) \mathrm{CT}_{\text {grain }}+\phi \mathrm{CT}_{\text {water }} .
$$

Pixels of the partially saturated maps have CT values (see Figure 3c) given by

$$
\mathrm{CT}_{\text {part sat }} \approx(1-\phi) \mathrm{CT}_{\text {grain }}+\phi\left(v_{2} \mathrm{CT}_{\text {water }}+v_{1} \mathrm{CT}_{\text {gas }}\right),
$$

where $\mathrm{CT}_{\text {water }}$ and $\mathrm{CT}_{\text {gas }}$ are the $\mathrm{CT}$ values of water and nitrogen and $v_{1}$ and $v_{2}$ are the percentages of water and gas occupying the pore space, respectively. To increase contrast, an X-ray dense solute is often added to experimental fluids. In these experiments, sodium iodide was dissolved in the water to $5 \%$ by weight.

Essentially, equations 1-3 assume that the CT value of a pixel at mesoscale resolution is related linearly to the CT values of its constituents in proportion to volume fractions. Thus, for large differences in CT values, which may occur at interfaces between mineral grains and pore space, these approximations can have considerable error. Ketchom and Carlson (2001) suggest the errors can be ignored because mineral-grain interfaces are rotated randomly relative to the

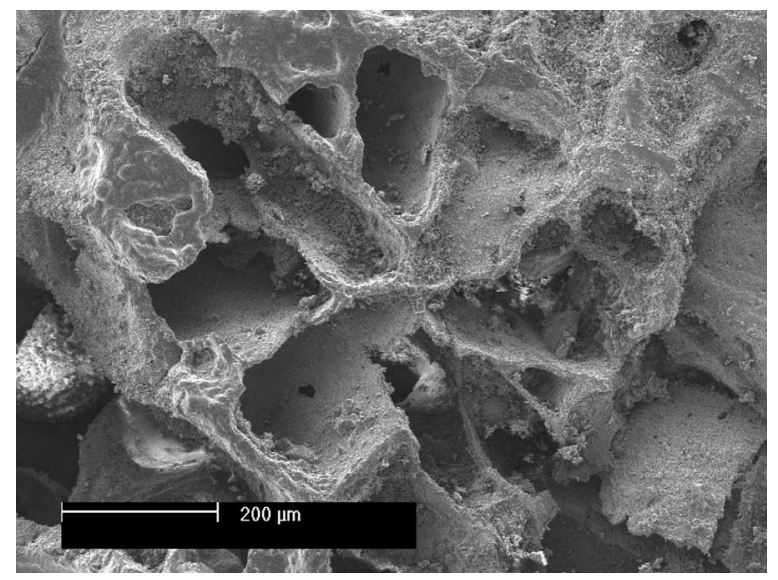

Figure 2. Higher-resolution SEM image of a typical MGL sample having no fluid saturation. 
plane of the scan; however, when the interfaces are parallel to the plane of the scan, errors can be as high as $10 \%$.

In general, the CT value (at mesoscale resolution) does not change greatly as the relative percentage of gas to water in the pore space alters. The change in CT value associated with changes in the percentage of gas saturation is much less than the CT values of the rock frame itself. Thus, to identify regions of the core sample that contain gas, the processing procedure of Cadoret et al. (1995) is implemented. Cadoret's procedure involves creating three different types of maps:

1) Porosity map $=\{$ fully water saturated image $\}-\{$ dry image $\}$

2) Gas-content map $=\{$ fully water saturated image $\}-\{$ partially water saturated images\}

3) Gas-saturation map = Gas-content map/porosity map

The pixels in each of the created maps have CT values as follows:

$$
\begin{aligned}
& \mathrm{CT}_{\text {porosity }}=\mathrm{CT}_{\text {dry }}-\mathrm{CT}_{\text {full sat }}=\phi\left(\mathrm{CT}_{\text {water }}-\mathrm{CT}_{\text {air }}\right), \\
& \mathrm{CT}_{\text {gas cont }}=\mathrm{CT}_{\text {full sat }}-\mathrm{CT}_{\text {part sat }}=\phi v_{1}\left(\mathrm{CT}_{\text {water }}-\mathrm{CT}_{\text {gas }}\right),
\end{aligned}
$$

$$
\mathrm{CT}_{\text {gas sat }}=\frac{\mathrm{CT}_{\text {gas cont }}}{\mathrm{CT}_{\text {porosity }}}=\frac{v_{1}\left(\mathrm{CT}_{\text {water }}-\mathrm{CT}_{\text {gas }}\right)}{\left(\mathrm{CT}_{\text {water }}-\mathrm{CT}_{\text {air }}\right)} .
$$

These maps are displayed in Figure 3d-f. In theory, equation 4 can be used to estimate spatial fluctuations in rock porosity (in addition to the average rock porosity). However, it cannot be utilized in the present study because the CT value of the doped water was not measured.
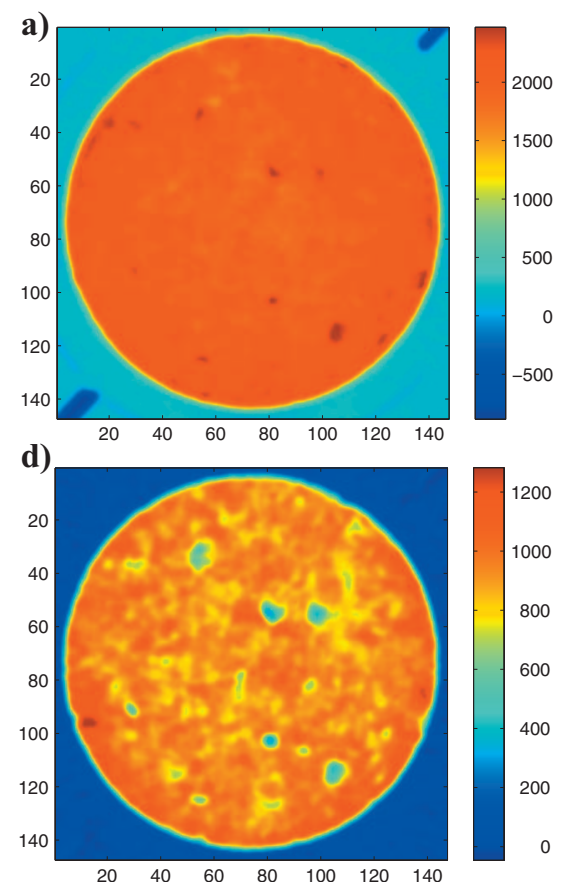
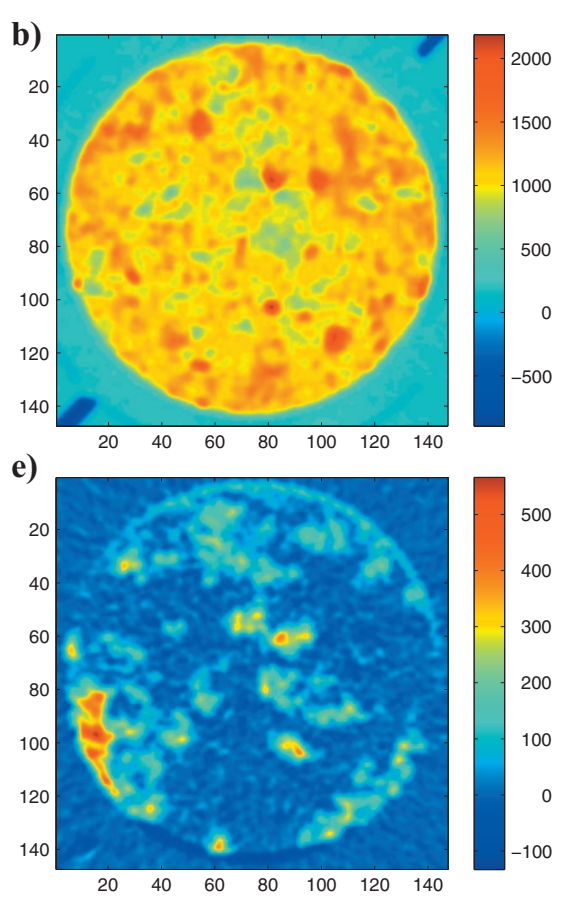

\section{Binary gas-saturation maps}

Determination of the percentage of gas saturation of each pixel comes from equation 6 , where we assume the CT value of nitrogen gas is close to the CT value of air. If this assumption is accepted, then maps generated using equation 6 show the spatial location of gassaturating pores and the percentage of gas saturation in those pores. Pixel values range from zero to one, where zero indicates fully water-saturated pores and one indicates fully gas-saturated pores; intermediate pixel values indicate the pore space is of mixed gas-water composition. The average gas saturation $\left\langle v_{1}\right\rangle$ of the core sample is calculated from the gas saturation of each pixel by

$$
\left\langle v_{1}\right\rangle=\frac{1}{N} \sum_{i=1}^{N} v_{1}(i)
$$

where $N$ is the total number of pixels.

To convert the images into binary media (necessary to extract certain types of quantitative statistics), a simple global-threshold technique is applied. Pixels having CT values below a certain threshold value are designated as water-saturated pixels; pixels having CT values above the threshold are counted as gas-saturated pixels. The threshold value is the mean CT value of the statistical distribution of pixel CT values. Thus, the threshold value represents the $C T$ value of an average pixel that contains some percentage of gas and water. Pixels having CT values below (or above) the threshold are understood to contain less (or more) gas relative to the average pixel.

In Figure 4a-i, the gas-saturation maps are shown for the drainage experiment on MGL 2a (only the inner square of the core sample is shown). During the initial stages of the experiment (Figure $4 a$ and $b$; gas saturation is less than 20\%), distinct patches of gas-bearing pores exist. Once average gas saturations (Figure 4c-i) exceed $20 \%$, distinct clusters of gas-bearing pores are no longer visible.

Figure 3. (a) X-ray images of dry core; (b) fully water-saturated core; (c) partially water-saturated core; (d) porosity map; (e) gas-content map; and (f) gas-saturation map. 
In Figure 5, the gas-saturation map (left) is compared with its corresponding binary map (right). It shows that applying a threshold around the mean produces a binary map that preserves the main features of the full gas-saturation map. This is true for most scans; however. more elaborate thresholding techniques such as indicator kriging could be used (Oh and Lindquist, 1999).

\section{STATISTICAL ANALYSIS OF BINARY MAPS AND CORRELATION PROPERTIES}

The drainage experiment proceeds, and the saturation maps (Figure $4 \mathrm{a}-\mathrm{i}$ ) become more complicated as more of the pore space becomes affected by the presence of gas. To characterize the fluid distribution (and changes in fluid distribution) of each mesoscale image, descriptive statistics (such as correlation function and correlation length) are extracted from the binary images using Monte Carlo methods. By comparing statistics derived from each map, it is possi- ble to characterize changes in fluid distribution caused by changes in gas saturation.

\section{Indicator functions}

For each binary map, an indicator function is defined that describes the segmentation of the map into different subdomains (Torquato, 2002). The indicator function is defined as

$$
I^{(i)}(r ; \omega)=\left\{\begin{array}{ll}
1 & \text { if } r \in V_{i}(\omega) \\
0 & \text { otherwise }
\end{array},\right.
$$

where $I^{(1)}$ is the indicator function for the gas-saturated domain $V_{1}$ and $I^{(2)}$ is the indicator function for the water-saturated domain $V_{2}$.

The role of the indicator function can be found by considering all points $r_{g}=\left\{(x, y):(x, y) \in V_{1}\right\}$ belonging to the gas-saturated domain and the values assumed by the different indicator functions. For this set of points, the indicator function for the gas-saturated do-
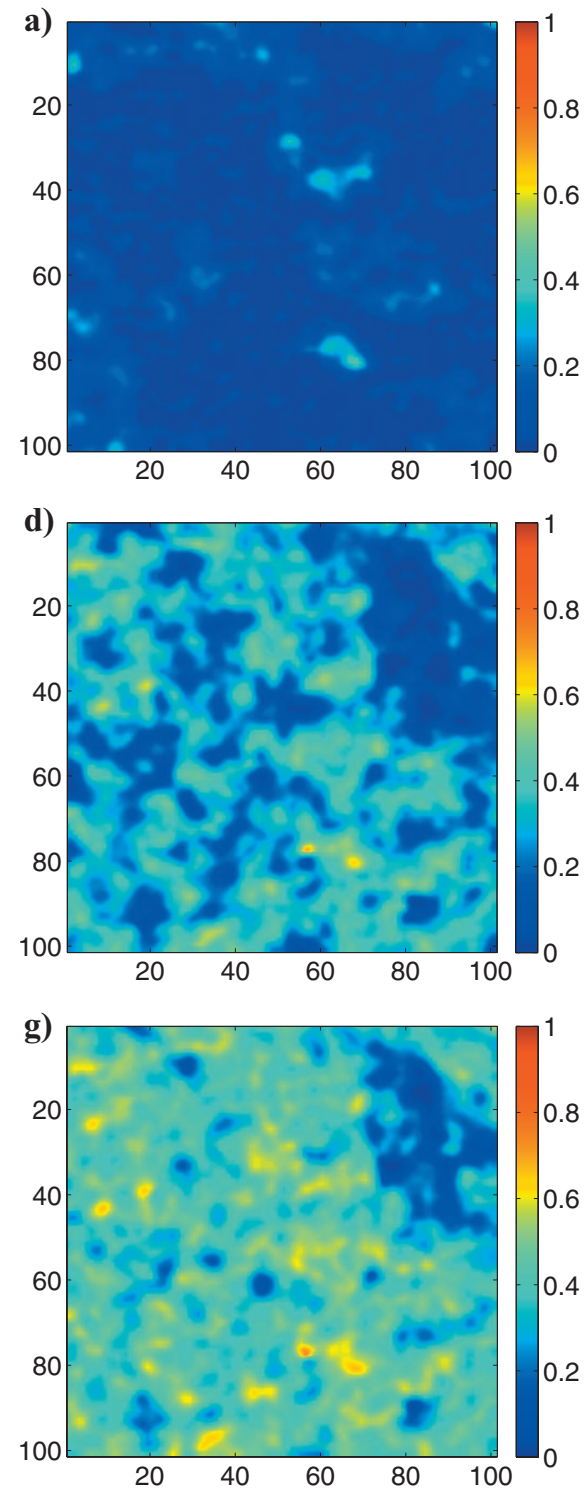
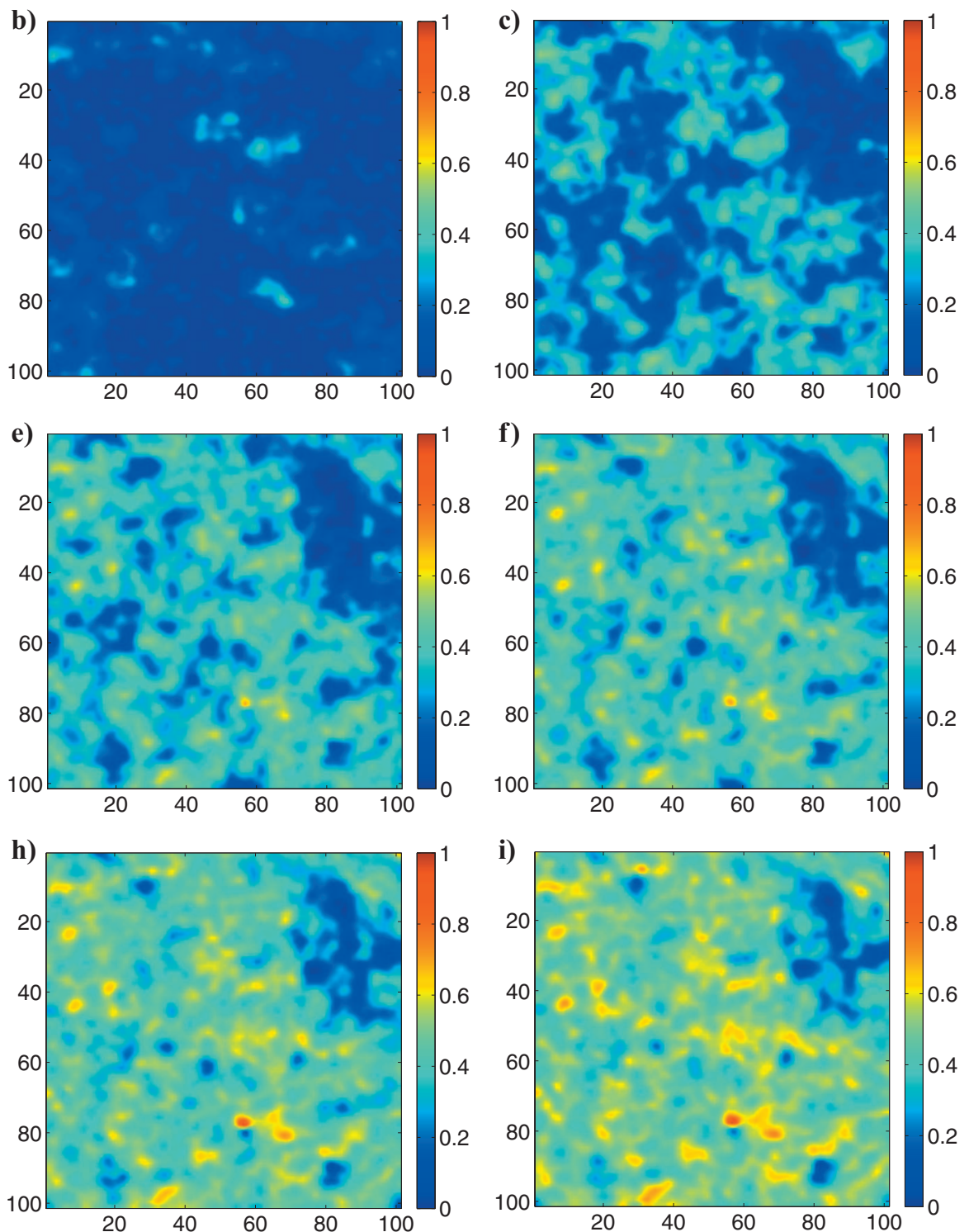

Figure 4. Gas-saturation maps at different stages of the injection experiment. Dark blue means zero gas saturation; dark red means full gas saturation. 
main $I^{(1)}\left(r_{g}\right)=1$; the indicator function for the water-saturated zone $I^{(2)}\left(r_{g}\right)=0$. For all points belonging to the water-saturated domain,the reverse is true. Hence,

$$
I^{(1)}(r)+I^{(2)}(r)=1 \forall r=(x, y) \in V=V_{1} \cup V_{2} .
$$

The average of either indicator function $I^{(i)}(r)$ is equal to the volume concentration of that domain: $\left\langle I^{(i)}\right\rangle=\varphi_{i}$. This is equivalent to the probability $P$ that a randomly chosen point on the map will belong to the gas-saturated (or water-saturated) domain. In other words, $\left\langle I^{(1)}\right\rangle$ $=P\left\{I^{(1)}(r)=1\right\}=\varphi_{1}$ and $\left\langle I^{(2)}\right\rangle=P\left\{I^{(2)}(r)=1\right\}=\varphi_{2}$. It is not necessarily the case that the volume concentration of the gas-saturated domain is equal to the average gas saturation of the partially saturated core sample, or $\left(\varphi_{1} \neq v_{1} \therefore \varphi_{2} \neq v_{2}\right)$.

\section{Autocorrelation functions and correlation length}

The autocorrelation function $\chi(r)$ of the binary map is related to the indicator functions of the gas- and water-saturated domains by

$$
\begin{aligned}
\chi(d r)= & \left\langle I^{(1)}(r)-\varphi_{1}\right\rangle\left\langle I^{(1)}(r+d r)-\varphi_{1}\right\rangle=\left\langle I^{(2)}(r)-\varphi_{2}\right\rangle \\
& \times\left\langle I^{(2)}(r+d r)-\varphi_{2}\right\rangle
\end{aligned}
$$

where $d r$ is the scalar distance separating two points (the correlation lag). Equation 10 is appropriate for isotropic maps, where $\chi(r)$ does not vary with line orientation. Because the indicator function of each
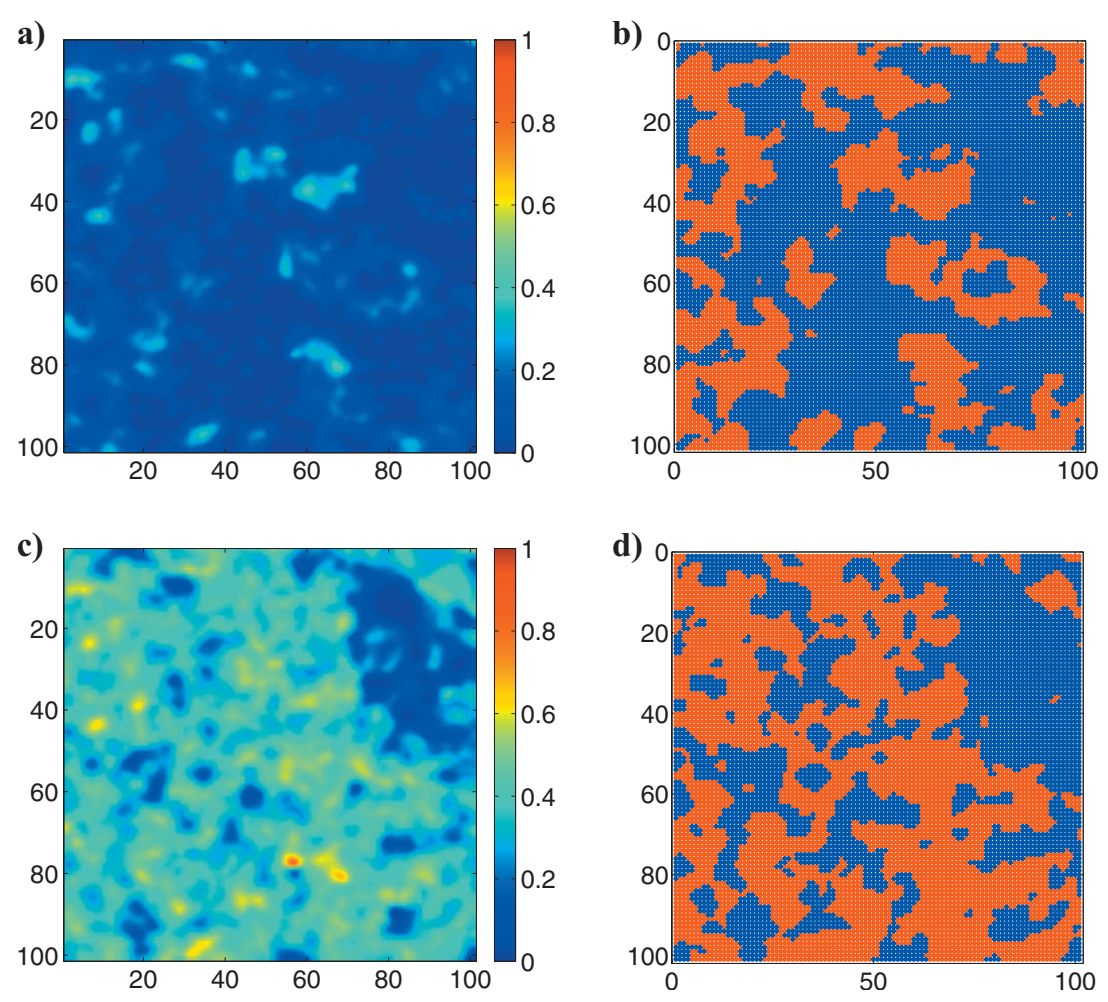

Figure 5. Example of global thresholding applied to gas-saturation maps of (a) scan 11 and (b) scan 15 to create the corresponding binary maps (c) and (d). domain is equivalent to the probability of a random point residing in that domain, the correlation function can be determined from

$\chi(r)=S^{(1)}(r, r+d r)-\varphi_{1}^{2}=S^{(2)}(r, r+d r)-\varphi_{2}^{2}$,

where $S^{(1)}(r, r+d r)$ and $S^{(2)}(r, r+d r)$ are the two-point probability functions for the gas- and water-saturated domains, respectively.

The two-point probability function refers to the probability that two random points displaced by a scalar distance $d r$ reside in the same domain. Thus, $S^{(1)}(r, r+d r)$ is the probability that two random points will reside in the gas-saturated domain and $S^{(2)}(r, r$ $+d r)$ is the probability that two random points will reside in the water-saturated domain. Clearly, as the distance separating the points reduces to zero, the two-point probability functions reduce to onepoint probability functions accordingly:

$\lim _{d r \rightarrow 0} S^{(i)}(r, r+d r)=S^{(i)}(r, r)=S^{(i)}(r)=\varphi_{i}$.

Moreover, if no long-range order exists, the two-point probability function converges to

$$
\lim _{d r \rightarrow \infty} S^{(i)}(r, r+d r)=\varphi_{i}^{2} .
$$

Substituting equations 12 and 13 into equation 11 shows that the correlation function has limiting values of $\chi(0)=\varphi_{1} \varphi_{2}$ and $\chi(\infty)=0$.

To determine the correlation function for each binary map, we extract the two-point probability function using Monte Carlo methods. From equation 11, it is clear that the correlation function can be determined using two-point probability functions for either the gas- or water-saturated domain. We choose to extract the two-point probability function corresponding to the gas-saturated domain. The two-point probability function of the water-saturated domain can be calculated from the two-point probability function for the gas-saturated domain using

$$
\begin{aligned}
S^{(2)}(r, r+d r)= & S^{(1)}(r, r+d r)-\varphi_{1}^{2} \\
& +\varphi_{2}^{2} \\
= & S^{(1)}(r, r+d r)+1 \\
& -2 \varphi_{1} .
\end{aligned}
$$

The one-point probability function for the gassaturated domain is determined by randomly sampling the image at one point and recording the times that one point corresponds to the gas-saturated domain. This total is divided by the points sampled to give the one-point probability density function. It is equivalent to the volume fraction of the gas-saturated domain in the binary map.

The two-point probability density function of the gas-saturated domain is obtained by randomly sampling the image at two points separated by scalar distances $d r$. A record is made of the times both sample points simultaneously reside in the gas-saturated domain for each scalar distance $d r$. To determine the two-point probability density function, the record is divided by the times the binary map is sampled at each scalar distance. 


\section{Correlation function versus relative percentage fluid saturation}

Correlation functions are extracted from the maps of sample MGL 2a. The objective is to assess whether the correlation function is sensitive to changes in the percentage of fluid saturation. By visual inspection of the shape of the correlation functions, two predominant sets are identified (Figure 6). The first set contains correlation functions that have an exponentially decreasing appearance; the second set contains correlation functions of a different shape.

In Figure $6 a$, the first group of correlation functions is plotted. The relative percentage of fluid saturation is shown in the legend (gas saturations $<23 \%$ ). There are only small observable differences in each correlation function. Also plotted is the average of these correlation functions (thick dashed line), obtained by taking the mean of this group. The unnormalized variance is shown in Figure 6d (dotted line with circles). In Figure 6b, the second group of correlation functions are plotted (gas saturations $>23 \%$ ). There are no observable differences in each correlation function. The average correlation function of this set (thick dashed line) is plotted, and the variance is shown in Figure 6d (dotted line with crosses). Comparison of the average correlation functions for each group (Figure 6c) shows that the shape of each curve is different.

On the basis of Figure $6 \mathrm{a}-\mathrm{d}$, it appears that correlation functions are only sensitive to changes in fluid saturation below a critical percentage (here, approximately 23\%). Above this value, there are only minor changes in the correlation function as gas saturation is increased (the variance shows that small changes occur around the Debye correlation length, as explained below). However, at greater $\mathrm{X}$-ray tomography resolutions, the correlation functions may show an increased dependence on gas saturation (when percentages are high).

\section{Correlation function versus rock type}

Percentage fluid saturation influences the shape of the correlation function (Figure 6a-d), but it is not the only influencing factor. In Figure $6 \mathrm{e}$ and $\mathrm{f}$, the average correlation function and variances (for gas saturations greater than the critical percentage) are shown for three different types of rocks: MGL 8 (crosses), MGL 6 (circles), and MGL 2a (triangles). The average correlation functions (Figure 6e) differ in shape.

Because the average correlation functions for each rock type are obtained from maps having gas saturations greater than the critical percentage, the shape differences are not caused by different percentages of gas. Rather, it is a consequence of each rock having different fluid-transport properties, i.e., porosity and permeability variations (wettability characteristics do not play a role here because all rocks are composed of the same type of mineral grain). Unfortunately, experimental measurements of rock porosity and permeability are not known for these rock samples.

\section{Debye and mean correlation lengths}

A host of different types of length scales can be derived from a correlation function. Two common ones are the Debye and meanlength scales. The first length scale can be derived from the correlation function by assuming that the binary map is a Debye random material (Debye and Bueche, 1949). For structures in which one phase consists of random shapes and sizes, the correlation function obeys

$$
\chi(r)=\exp \left(\frac{-|r|}{a}\right)
$$

where $a$ is the Debye correlation length, defined as $\chi(a)=1 / e$.

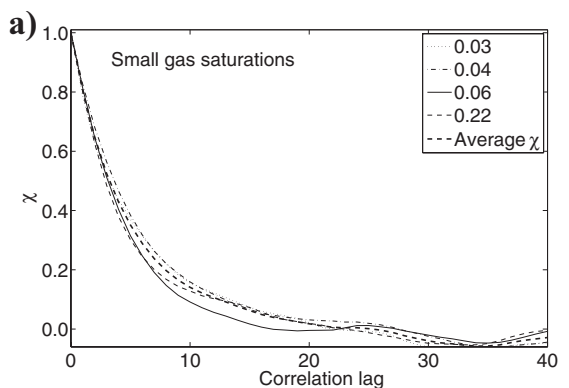

d)

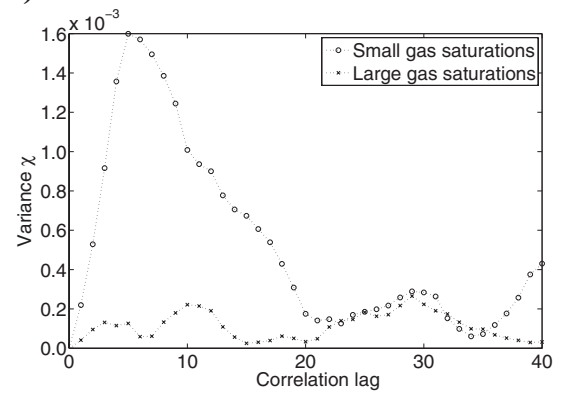

b)

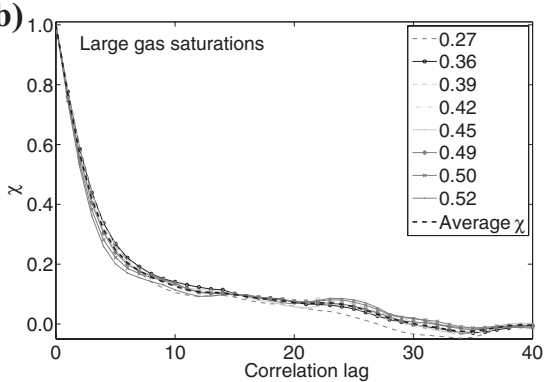

e)

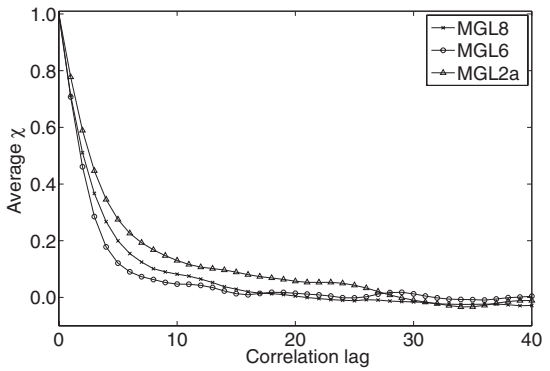

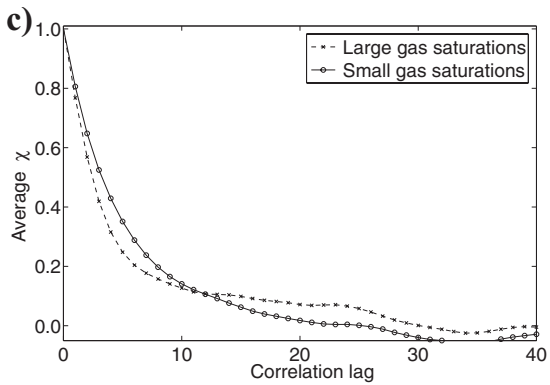

f)

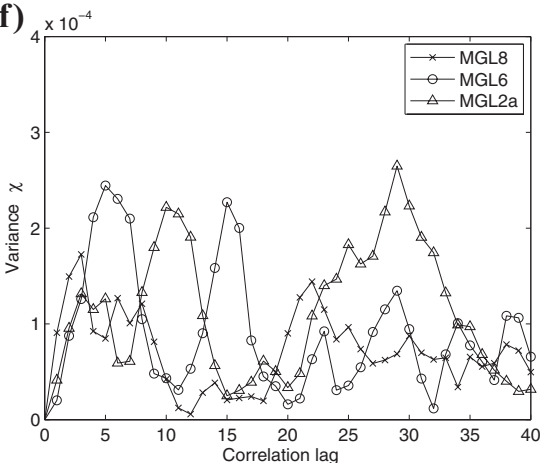

Figure 6. (a, b) Correlation functions for different scans for the MGL 2a sample. (c-f) Averages and variances of $\chi(r)$ for samples MGL 2a, MGL 6 , and MGL 8. The correlation lag is given in number of pixels. 
The second length, termed the mean length, is derived from the correlation function as

$$
l_{m}=\left\{\int_{0}^{\infty} r \chi(r) d r\right\}^{1 / 2} .
$$

This length scale arises in rigorous bounds on the fluid permeability and trapping constant of 3D isotropic random porous media (Berryman and Blair, 1986; Torquato, 2002). If the correlation function in equation 11 is an exponentially decreasing function, the mean length is equal to the Debye length $l_{m}=a$.

Any length scale derived from a correlation function produces a length scale representative of the average spatial properties of the medium (a consequence of equation 11). That is, the Debye or meanlength scales are measures of how coarse or fine the material is, rather than indicating an average size of either phase. Thus, either length scale (defined in equation 15 or 16) derived from the correlation function will not indicate the size of the gas or water patches in the binary map.

In Figure 7, the Debye correlation length is extracted from the correlation functions of MGL 2a (circles), MGL 6 (asterisks), and MGL 8 (crosses). The Debye correlation length of MGL 2a decreases approximately linearly as gas saturation increases. The Debye length of MGL 8 also decreases approximately linearly with saturation, and a similar trend can be observed with MGL 6. Noting that, the variances extracted in the previous section indicate that small changes in the correlation functions occur around the Debye correlation length. Thus, these results are consistent with the observation that the correlation function is mostly insensitive to the percentage of fluid saturation at large gas saturations.

By analyzing the mean correlation functions for MGL 2a at both low and high gas saturations, an interesting observation can be made. It appears that the mean correlation function for small gas saturations (solid line) is approximated well by a Debye correlation function (dotted line) with correlation length of $a=3.5$ (see Figure 8). On the other hand, at high gas saturations, there is a good corre-

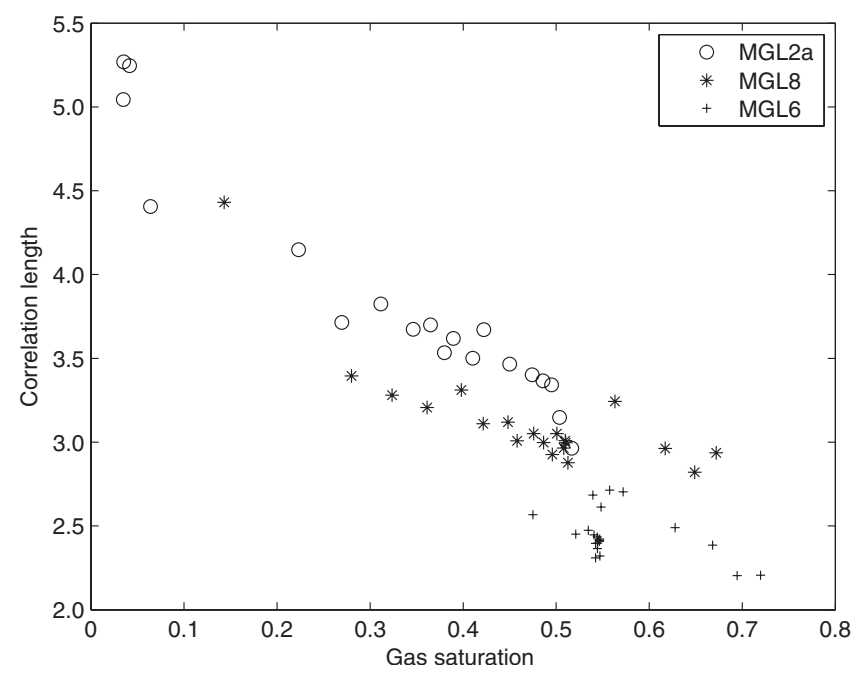

Figure 7. The Debye correlation length for different gas saturations for samples MGL 2a, MGL 6, and MGL 8. The Debye correlation length is given in number of pixels. spondence only between the mean correlation function (dashed line) and the Debye correlation function (dashed-dotted line) at small offsets.

In Figure 9, the mean correlation function for large gas saturations (MGL 2a) is approximated well by two Debye correlation functions: one that models small-offset behavior $\chi_{\text {small }}$ and one that models large-offset behavior $\chi_{\text {large }}$ :

$$
\begin{aligned}
\chi_{2}(r) & =\chi_{\text {small }}+\chi_{\text {large }} \\
& =b_{S} \exp \left(-\frac{r}{a_{S}}\right)+b_{L} \exp \left(-\frac{r}{a_{L}}\right) \\
& =b_{S} \exp \left(-\frac{r}{a_{S}}\right)+\left(1-b_{S}\right) \exp \left(-\frac{r}{a_{L}}\right),
\end{aligned}
$$

where $b_{S}, b_{L}$ are weighting coefficients of the correlation functions

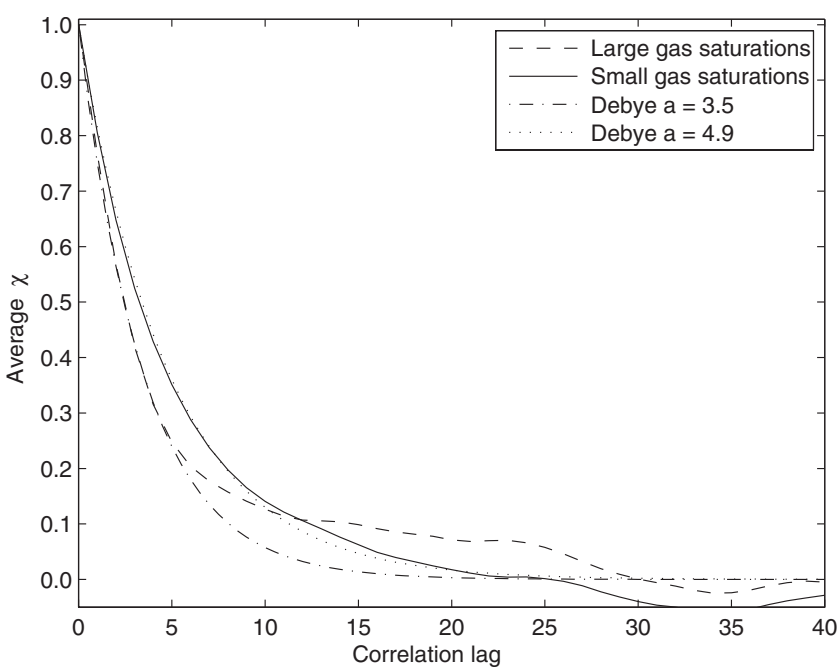

Figure 8 . The Debye correlation function having different correlation lengths (given in number of pixels) compared against the average correlation functions of MGL 2a. The correlation lag $r$ is also given in number of pixels.

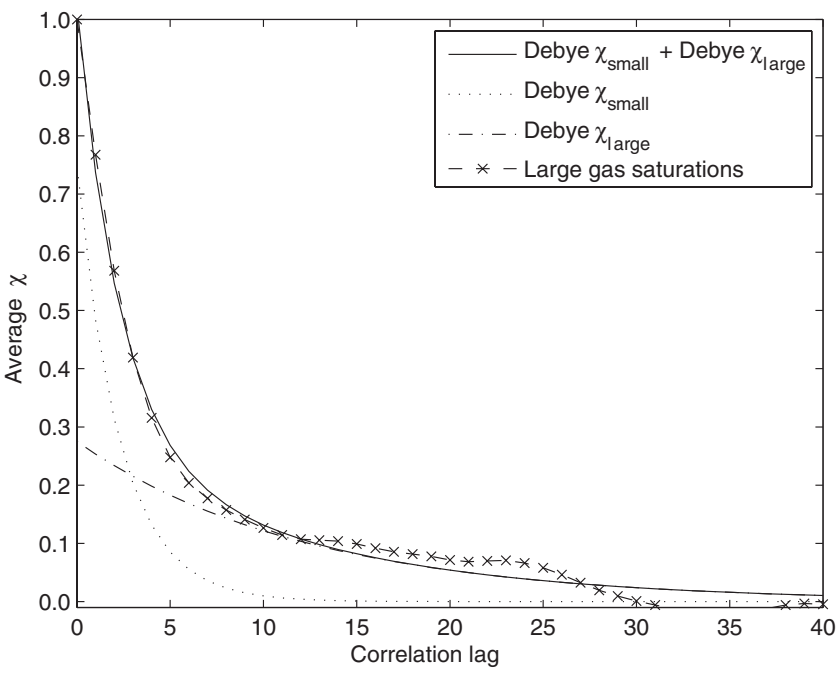

Figure 9. The average correlation function at large gas saturations is approximated by two Debye correlation functions $\chi_{\text {small }}$ and $\chi_{\text {large }}$. 
(with $1>b_{S}>b_{L}>0$ ), and where $a_{S}, a_{L}$ are the usual Debye correlation lengths (with $a_{L}>a_{S}>0$ ). In Table 1, parameters specifying $\chi_{2}(r)$ according to equation 17 are given for the mean correlation functions for large gas saturations of samples MGL 2a, MGL 6, and MGL 8.

On the basis of the statistical analysis presented here, three conclusions can be made regarding fluid distribution versus saturation. First, the correlation length decreases linearly as gas saturation is increased. Second, for small gas saturations, say, $v_{1} \leq 20 \%$, the correlation function can be well approximated by a single Debye correlation function. Third, for large gas saturations, say, $v_{1} \geq 20 \%$, the correlation function can be approximated reasonably well by a superposition of two weighted Debye correlation functions.

\section{MODELING P-WAVE ATTENUATION AND PHASE-VELOCITY DISPERSION}

A number of theoretical approaches (White, 1976; Gist, 1994; Mavko and Mukerji, 1998; Johnson, 2001; Müller and Gurevich, 2004) aim at modeling the physical effect that partial fluid saturation has on compressional-wave propagation. These studies reveal that for low-frequency seismic waves, there is enough time for induced fluid pressures to equilibrate at a constant pressure. In this quasi-static limit, the saturation can be considered homogeneous; hence, the rock can be viewed as saturated by a homogeneous mixture of the fluids. For this case, the bulk modulus of the saturated rock can be determined from the Gassmann equation using an effective fluid bulk modulus $K_{f}$ given by Wood's formula (saturation-weighted harmonic average of the bulk moduli of fluids $K_{f 1}, K_{f 2}$ ):

$$
K_{f}=\left(\frac{S_{1}}{K_{f 1}}+\frac{S_{2}}{K_{f 2}}\right)^{-1} .
$$

This is known as the Gassmann-Wood limit. However, fluid-pressure equilibration throughout the rock will occur only for very low frequencies or very small patches. Specifically, it occurs when the characteristic patch size is small compared to the fluid diffusion length (Norris, 1993):

$$
\lambda_{d}=\sqrt{\frac{\kappa N}{\omega \eta}},
$$

where $\kappa$ is the rock permeability, $\eta$ the fluid viscosity, $\omega$ the angularwave frequency, and $N$ is a combination of poroelastic moduli specified below.

On the other hand, when wave frequencies are sufficiently high (or there are very large patch sizes greater than diffusion length), there is insufficient time for fluid-pressure equilibration to occur. Hence, there are individual patches throughout the rock having constant but different fluid pressures. For this circumstance, Gassmann's equation can be applied to individual patches to compute the saturated bulk modulus of each patch. Moreover, because the saturated shear modulus $\mu$ is independent of the fluid bulk modulus, Hill's theorem can be used to determine the overall saturated bulk modulus $K_{H}$ :

$$
\begin{aligned}
\left(K_{H}+\frac{4}{3} \mu\right)^{-1}= & S_{1}\left(K_{1}+\frac{4}{3} \mu\right)^{-1} \\
& +S_{2}\left(K_{2}+\frac{4}{3} \mu\right)^{-1},
\end{aligned}
$$

where $K_{1}$ and $K_{2}$ are the saturated bulk moduli according to Gassmann's theory for each fluid patch. This is known as the GassmannHill limit.

At intermediate wave frequencies (or intermediate patch sizes), uneven deformation of fluid patches by the passing wave results in wave-induced fluid flow, which causes wave attenuation and velocity dispersion. This is the physical picture we seek to model for realistic fluid distributions obtained from tomographic images of partially saturated rock. At present, there is only one patchy saturation model (detailed below) that can take in spatial information obtained from tomographic images.

\section{The continuous random media model of patchy saturated rocks}

P-wave attenuation and velocity are modeled using the CRM for patchy saturated rocks (refer to Toms et al., 2006, 2007). This model assumes that rock properties such as porosity, permeability, dryframe bulk modulus, and grain bulk modulus are constant throughout the rock. In other words, only heterogeneities in fluid bulk modulus are considered. This model is a simplification of a more general model developed by Müller and Gurevich (2005), which estimates attenuation and phase velocity for porous rocks that contain heterogeneities in frame and fluid properties. However, to predict attenuation and velocity dispersion from tomographic images of drainage experiments, we use the simplified patchy-saturation version, assuming either the porous rock used in the experiment is homogeneous (except for its saturating fluid) or the estimated velocities are affected more greatly by fluid heterogeneities than by heterogeneities in other properties (such as porosity and permeability).

In the CRM approach, the saturated $P$-wave modulus $\widetilde{H}$ (after rearranging equation 15 of Toms et al., 2007) can be expressed as

$$
\tilde{H}(\omega)=H^{W}\left(1+\delta\left[\tau \xi^{2}+(\tau-1) \xi\right]\right),
$$

where the complex, frequency-dependent function $\xi$ is

$$
\xi(\omega)=k_{s \mathrm{P}}^{2} \int_{0}^{\infty} r B_{M}(r) \exp \left[i k_{s} \mathrm{P} r\right] d r,
$$

Table 1. Parameters specifying $\chi_{2}(r)$ according to equation 17 are given for the mean correlation functions for large gas saturations of samples MGL2a, MGL6, and MGL8. The Debye correlation length is given in number of pixels (pixel length $\cong 0.36 \mathbf{m m}$ ). The last column shows the rms error of the fit.

\begin{tabular}{lccrcc} 
Sample & $a_{S}$ & $b_{S}$ & $a_{L}$ & $b_{L}$ & rms error \\
\hline MGL2a & 2.30 & 0.73 & 12.28 & 0.27 & 0.02234 \\
MGL6 & 2.02 & 0.93 & 12.58 & 0.07 & 0.01357 \\
MGL8 & 2.77 & 0.85 & 9.11 & 0.15 & 0.01153 \\
\hline
\end{tabular}


involving the slow P-wavenumber $k_{s \mathrm{P}}=\sqrt{i \omega \eta / \kappa N}$ and the normalized (unit variance) correlation function of the pore-space modulus $B_{M}(0)=1$ (further properties of the function $\xi(\omega)$ are discussed in Müller et al., 2007). In equation $18, H^{W}$ denotes the $\mathrm{P}$-wave modulus in the Gassmann-Wood limit and

$$
\delta=\frac{\left(H^{H}-H^{W}\right)}{H^{W}},
$$

where $H^{H}$ is the P-wave modulus in the Gassmann-Hill limit. Equation 23 ensures that the model converges to the theoretical limits given by Gassmann-Wood and Gassmann-Hill relations.

The coefficient $\tau$ contains the variance of the fluid modulus fluctuations $\sigma_{M M}^{2}$ :

$$
\tau=\frac{\alpha^{2} N \sigma_{M M}^{2}}{(4 H)} .
$$

Hence, for small fluid contrasts, the variance $\sigma_{M M}^{2}$ is small and $\tau$ is negligible. This reduces equation 18 to

$$
\tilde{H}(\omega)=H^{W}(1-\delta \xi(\omega)) .
$$

In equations 18-21, we use the standard nomenclature of linear poroelasticity theory: The dry and saturated $\mathrm{P}$-wave moduli are $L=K$ $+4 / 3 \mu$ and $H=K^{\text {sat }}+4 / 3 \mu^{\text {sat }}$, respectively. Gassmann's equations relate dry and saturated bulk modulus, $K$ and $K^{\text {sat }}$, and dry and saturated shear modulus, $\mu$ and $\mu^{\text {sat }}$ via $K^{\text {sat }}=K+\alpha^{2} M, \mu^{\text {sat }}=\mu$. Here, $\alpha=1-K / K_{s}$, and $M=\left([\alpha-\phi] / K_{s}+\phi / K_{f}\right)^{-1}$ is called the fluid modulus. It is a function of the grain bulk modulus $K_{S}$, the fluid bulk modulus $K_{f}$, and the porosity $\phi$, and $N$ is defined as $N$ $=M L / H$. The bulk density $\rho$ is computed via the density of the grain material $\rho_{S}$ and the fluid density $\rho_{f}: \rho=(1-\phi) \rho_{S}+\phi \rho_{f}$. The poroelastic moduli $N$ and $H$ in equation 21 involve the saturationweighted average of the fluid modulus $\bar{M}=M_{1} \nu_{1}+M_{2} \nu_{2}$. Real and imaginary components of equation 18 yield the effective phase velocity

$$
v(\omega)=\sqrt{\frac{\operatorname{Re}\{\tilde{H}\}}{\rho}}
$$

and specific attenuation (inverse quality factor)

$$
Q^{-1}=\frac{-\operatorname{Im}\{\tilde{H}\}}{\operatorname{Re}\{\tilde{H}\}} .
$$

The CRM correlation function describes the spatial variation of the fluid modulus $M$, resulting from fluctuations in fluid bulk modulus. We apply the CRM model to a situation of continuous variation in fluid bulk modulus. This may arise when the pore space is saturated partially by two types of fluids, such that mesoscopic fluid heterogeneities appear as regions of rock partially saturated by different percentages of pore fluid (like irreducible water saturation). When this occurs, we can treat the patch as being saturated by an effective pore fluid having an effective fluid bulk modulus, which reflects the patch's mixed-fluid composition.

\section{Estimates of attenuation and velocity dispersion using statistical information}

The gas-saturation maps (Figure 4) show for a particular rock where gas-bearing pores exist. Specifically, the CT value of a pixel indicates the percentage of gas saturation in the pore space covered by that pixel (via equation 6). That is, pixel values range from zero to one, where they indicate $0 \%$ and $100 \%$ gas saturation, respectively, and intermediate values indicate pore space of mixed gas-water composition. Spatial statistics such as the correlation function or correlation length can be extracted from these maps. But, this information describes spatial characteristics of the percentage of gas variation on the mesoscale and not spatial information on the variation of fluid modulus M. So, it cannot be used directly in CRM theory.

We can, however, use information on the percentage of gas saturation to construct a new map that represents fluid bulk modulus variation on the mesoscale. This can be used to construct a fluid modulus map from which a correlation function consistent with CRM requirements can be extracted. In transforming the gas-saturation map into an effective fluid bulk modulus map, we assume that fluid pressures of gas and water in the porespace of each pixel are equal. This allows us to use Wood's fluid-mixing equation to construct an effective fluid bulk modulus for each pixel. However, in principle, it is also possible to utilize other fluid-mixing equations, such as Brie or Voigt averages, to create an effective fluid bulk modulus.

We propose the following procedure to model attenuation and dispersion using the CRM model. For step 1, convert the gas-saturation map into an effective fluid bulk modulus map $K_{f}^{W}$ using Wood's law:

$$
K_{f}^{W}=\frac{1}{\left[\frac{1-\mathrm{CT}_{\text {gas sat }}}{K_{f w}}+\frac{\mathrm{CT}_{\text {gas sat }}}{K_{f g}}\right]},
$$

where $\mathrm{CT}_{\text {gas sat }}$ is a particular gas-saturation map (see Figure 4 ), $K_{f w}$ is the fluid bulk modulus of water, and $K_{f g}$ is the fluid bulk modulus of gas.

For step 2, convert the fluid bulk modulus into the fluid modulus $M$ map using

$$
M=\frac{1}{\left[\frac{(\alpha-\varphi)}{K_{g}}+\frac{\varphi}{K_{f}^{W}}\right]} .
$$

Continuing with step 3 , calculate the mean of the fluid-moduli map using

$$
\langle M\rangle=\operatorname{mean}(M)=\frac{1}{N_{P}} \sum_{i=1}^{N_{P}} M_{i},
$$

where $N_{P}$ is the total number of pixels in the fluid-modulus map (the subscript $i$ refers to the $i$ th pixel). Also calculate the mean $\bar{M}$ of the fluid-modulus map using

$$
\bar{M}=\sum_{j=1}^{n} v_{j} M_{j}
$$

where $M_{j}$ is the fluid-modulus map divided into $n$ partitions of width $\Delta M$. That is, if $M_{1}=\min \left(M_{i}\right)$ and $M_{2}=\max \left(M_{i}\right)$, then $M_{2}-M_{1}$ $=n \Delta M$. Here, $v_{j}$ is the fraction of pixels having the same fluid modulus $M_{j}$. The partitioning of the fluid-modulus map is acceptable if $\bar{M}=\langle M\rangle$. The partitioning step is necessary to calculate the variance. 
For step 4, calculate the normalized variance of the fluid-moduli map using

$$
\sigma_{M M}^{2}=\frac{\sum_{j=1}^{n} v_{j}\left(M_{j}\right)^{2}}{\bar{M}^{2}}-1 .
$$

In step 5, calculate normalized centered autocorrelation function of fluid-moduli map. In step 6, calculate Wood's limit:

$$
\begin{aligned}
\frac{1}{M^{W}} & =\sum_{j=1}^{n} \frac{v_{j}}{M_{j}}, \\
H_{W} & =L+\alpha^{2} M_{W} .
\end{aligned}
$$

Step 7 calculates Hill's limit using

$$
\frac{1}{H^{H}}=\sum_{j=1}^{n} \frac{v_{j}}{L+\alpha^{2} M_{j}} .
$$

Computation of the quantities defined by equations 27-33, together with equation 21 , yields estimates of the frequency-dependent attenuation and dispersion for a particular gas-saturation map. Below we illustrate the technique for gas-saturation maps having small and large gas saturations.

Figure 10a shows the full gas-saturation map of scan 10, which has an average gas saturation of $4.6 \%$. Figure $10 \mathrm{~b}$ shows the effective-fluid/bulk-modulus map derived from the full gas-saturation map having assumed water and light gas-pore fluids. The color bar indicates the value of the fluid bulk modulus in pascals; regions that are dark red have an effective fluid bulk modulus close to water, and regions dominated by blue are affected by gas saturation. Figure $10 \mathrm{c}$ displays the fluid-modulus map calculated using equation 28. Figure $10 \mathrm{~d}$ compares correlation functions extracted from the gas-saturation map and fluid-modulus $M$ map.

An important point is illustrated here. The correlation functions in general are not interchangeable. That is, the correlation function extracted from the gas-saturation map is not equivalent to the correlation function extracted from the fluid-modulus $M$ map. There is one instance in which they are equivalent: when the contrast of the fluid bulk moduli is small. Figure 10e shows the modulus $M$ map, assuming water and heavy gas as pore fluids (for rock properties, see Table 2; for fluid properties, see Table 3). This map has a variance of $\sigma_{M M}^{2}$ $=0.0038$. Figure $10 \mathrm{f}$ shows the correlation function extracted from this map (dashed line). It coincides almost perfectly with the correlation function extracted from the gas-saturation map.

Figure 11 shows frequency-dependent attenuation and velocity dispersion calculated using two types of statistical information extracted from the fluid-modulus map in Figure 10d. The first approach centers on using the correlation function (Figure 10d) directly in equation 21, from which attenuation/velocity (dashed line with dots) can be estimated by taking real and imaginary components (equations 25 and 26). Clearly, this requires numerical integration of the correlation function. The second approach centers on approximating the correlation function in Figure 10d by an exponential correlation function (with Debye correlation length derived from the images). By making this approximation, we can use closed-form expressions for the saturated P-wave modulus (see Toms et al., 2006) to derive attenuation/velocity (solid line).
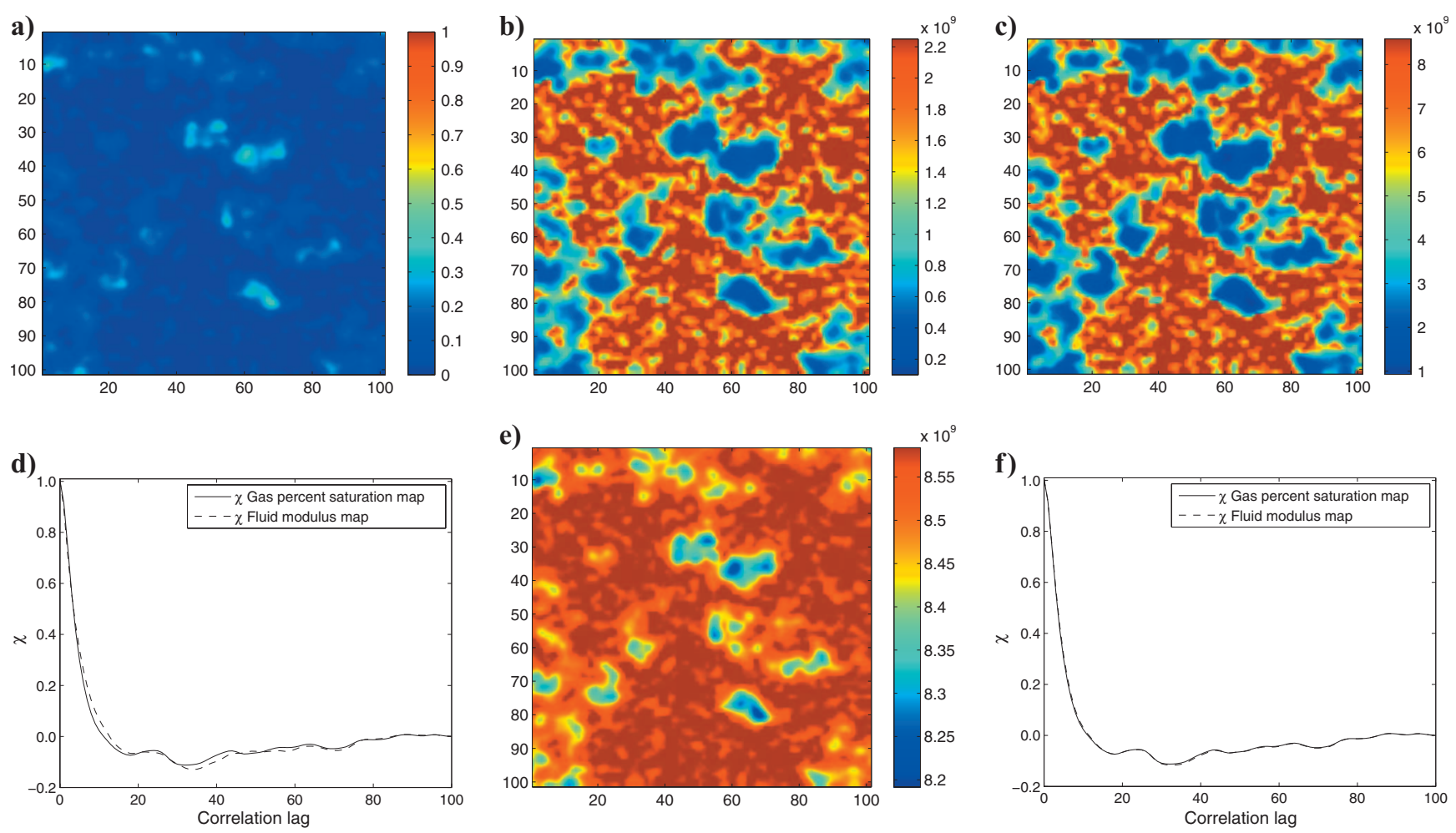

Figure 10. Based on scan 10: (a) gas-saturation map; (b) effective fluid bulk modulus map; (c) fluid-modulus map; (d) correlation functions extracted from the gas percentage fluid-saturation map (solid line) and from the fluid-modulus map (dashed line). The correlation functions obtained from each type of map are not equivalent; however, at low fluid-modulus contrast, they coincide, as illustrated in (e) and (f). 
Clearly, there is good correspondence between attenuation/velocity of both approaches when wave frequencies are low, as can be seen by the low-frequency asymptotes of attenuation (as shown in Figure 11a) and by convergence to Gassmann-Wood limits on velocity in Figure 11b. However, at higher frequencies the two approaches do not produce consistent attenuation and velocity estimates; this arises because the numerical integration routine utilized in the first approach becomes unstable at high frequencies, causing jagged frequency-dependent behavior for attenuation and velocity. However, the second approach is reliable over the entire frequency range.

Next, we perform the same analysis but for an average gas saturation of $34 \%$. Figure $12 \mathrm{a}$ shows the gas saturation map, Figure $12 \mathrm{~b}$ shows the effective-fluid/bulk-modulus map from which a fluidmoduli map is constructed, and Figure 12c shows the correlation function derived from the fluid-moduli map. Figure 13 shows frequency-dependent attenuation and velocity dispersion calculated using equations 21,25 , and 26 with numerical integration of the correlation function (Figure 12c) and by having approximated the correlation function with an exponent. There is good correspondence between attenuation and velocity dispersion at low frequencies; however, at high frequencies there are discrepancies. The discrepancies can be attributed to the breakdown of the numerical routine. Relative to the previous example (Figure 11), we see that the acoustic signatures become less pronounced at greater gas saturation.

\section{DISCUSSION}

Statistical analysis of X-ray tomography images provides useful information on fluid distribution and rock heterogeneity. Our analysis is restricted to the construction of the two-point probability func-

Table 2. Rock properties of Mount Gambier Limestone.

\begin{tabular}{cl}
\hline Property & Value \\
\hline$K$ & $26 \mathrm{GPa}$ \\
$\mu$ & $15 \mathrm{GPa}$ \\
$K_{g}$ & $74 \mathrm{GPa}$ \\
$\rho_{g}$ & $2650 \mathrm{~kg} / \mathrm{m}^{3}$ \\
$\phi$ & 0.25 \\
$\kappa$ & $5 \mathrm{e}-12 \mathrm{~m}^{2}$ \\
\hline
\end{tabular}

Table 3. Properties of pore fluids used in the numerical computations.

\begin{tabular}{cl}
\hline Property & Value \\
\hline$K_{f w}$ water & $2.25 \mathrm{GPa}$ \\
$K_{f}$ heavy gas & $0.25 \mathrm{GPa}$ \\
$K_{f}$ light gas & $0.1 \mathrm{GPa}$ \\
$\rho_{w}$ & $990 \mathrm{~kg} / \mathrm{m}^{3}$ \\
$\rho$ & $400 \mathrm{~kg} / \mathrm{m}^{3}$ \\
$\rho_{g}$ & $100 \mathrm{~kg} / \mathrm{m}^{3}$ \\
$\eta_{w}$ & $1 \mathrm{e}-3 \mathrm{~Pa} \mathrm{~s}$ \\
$\eta$ & $6 \mathrm{e}-5 \mathrm{~Pa} \mathrm{~s}$ \\
$\eta_{2}$ & $3 \mathrm{e}-5 \mathrm{~Pa} \mathrm{~s}$ \\
\hline
\end{tabular}

tion and autocorrelation function and its characteristic length scales. Higher-order statistical measures could be obtained in the same fashion. In particular, the so-called cluster correlation functions (see e.g. Torquato, 2002) might reveal additional information about the spatial connectivity of fluid patches.

Using fluid-distribution patterns and statistics obtained during saturation experiments on core samples to infer fluid-distribution patterns arising in the field (on reservoir scale) is a challenging task. However, from the laboratory we can gain small insights into which fluid processes, such as drainage, may produce certain fluid patterns in certain reservoir rocks. Hence, we might gain insight into which production techniques generate different types of reservoir-scale fluid distributions (see Sengupta and Mavko, 2003). It may also be possible to constrain reservoir flow simulations using statistical information such as the correlation function or length obtained from laboratory studies.

However, one must be very careful because transport properties (such as permeability) directly influence fluid flow and hence the generation of fluid-distribution patterns (and so the statistical description). In particular, one difficulty in extrapolating from the lab-

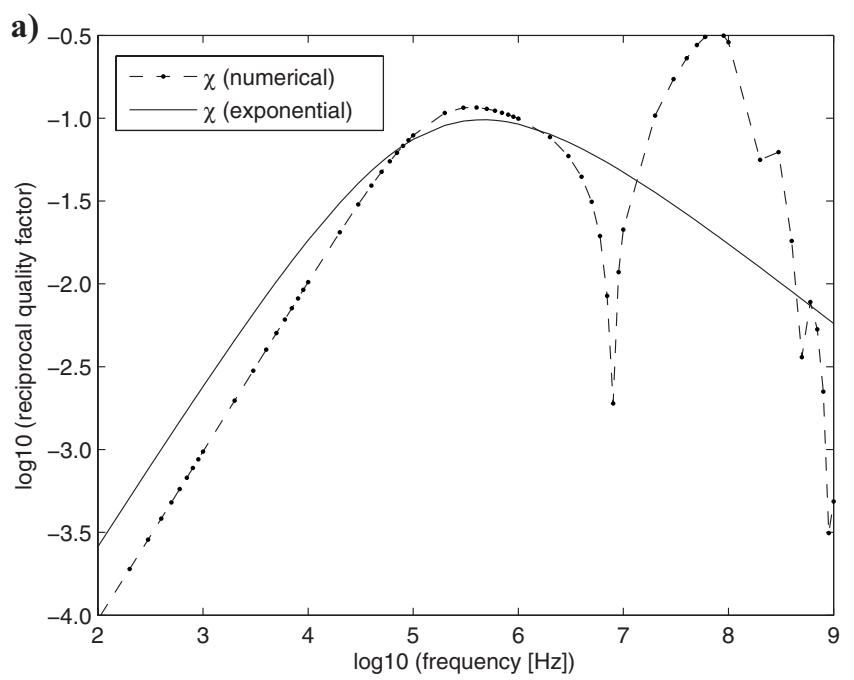

b)

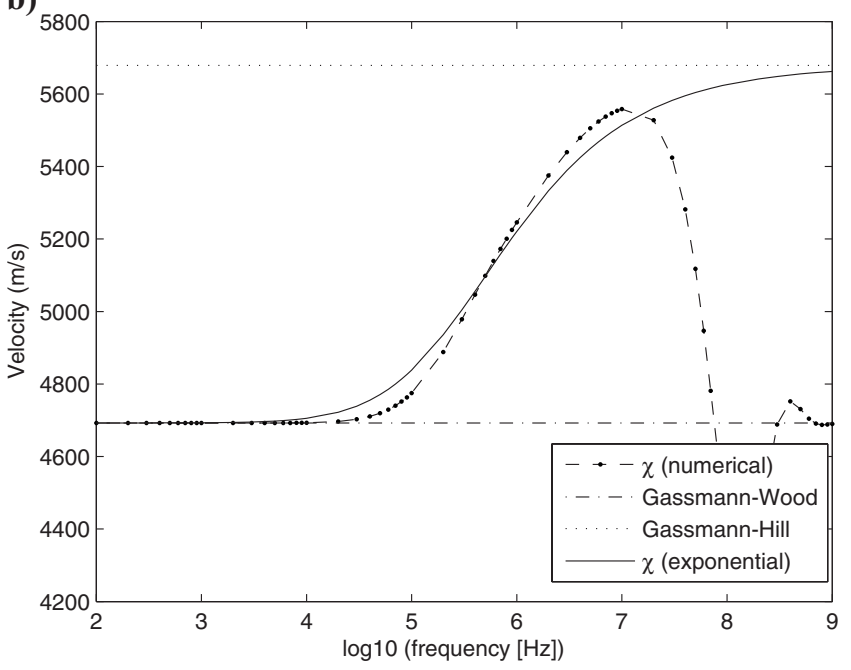

Figure 11. (a) Attenuation and (b) velocity from correlation functions extracted from gas-saturation map of scan 10 with average gas saturation of $4.1 \%$. 
a)

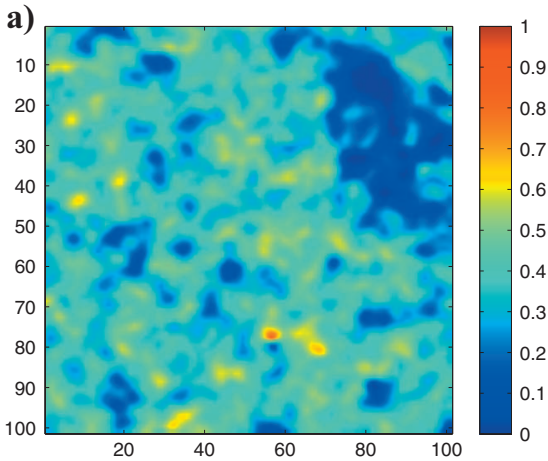

b)

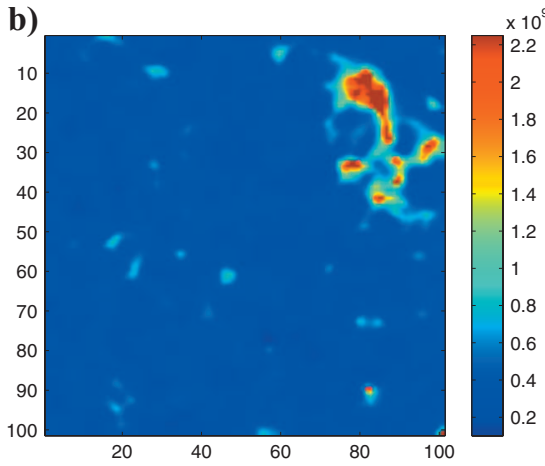

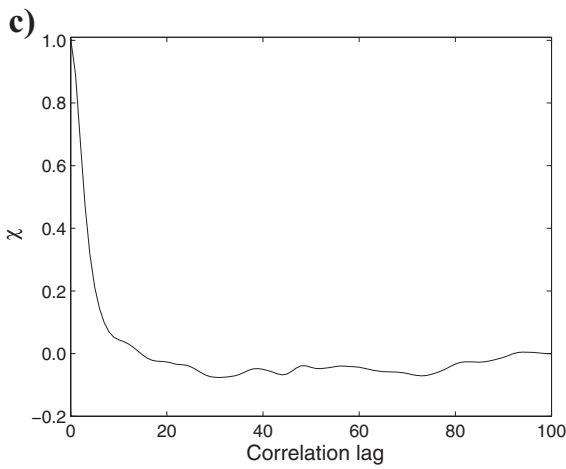

Figure 12. (a) Gas-saturation map; (b) effective bulk-fluid modulus map; and (c) correlation function for scan 15, with average gas saturation of $34.6 \%$.

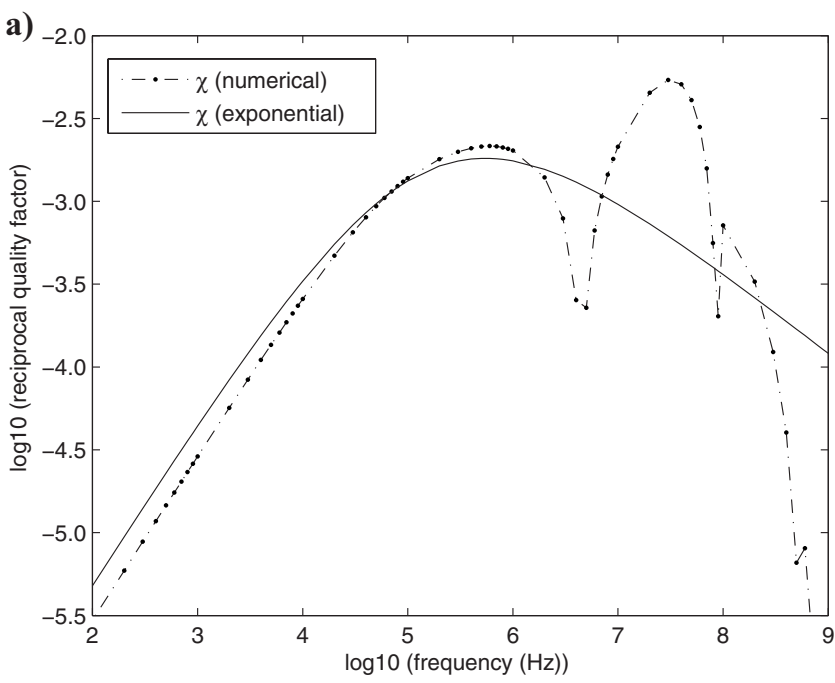

b)

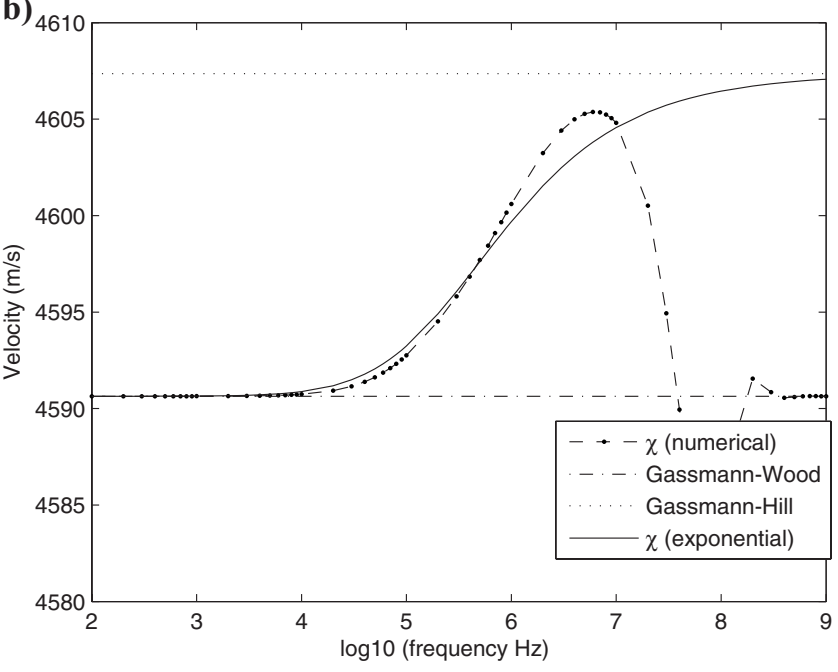

Figure 13. (a) Attenuation and (b) velocity from correlation functions extracted from the gas-saturation map of scan 15 with average gas saturation of $34.6 \%$.

oratory to the field lies in how we scale transport properties. As illustrated by Ehrenberg (2007), even laboratory measurements of porosity and permeability on 10-cm-diameter whole-core samples and on $2.5-\mathrm{cm}$ plugs extracted from each core sample can produce significantly different permeability estimates (see Neuman [1994] for a discussion on permeability scaling). Some questions to consider are (1) how the correlation length/function of a fluid distribution pattern is related to the transport properties of a rock and (2) how the descriptive statistics (such as the correlation length/function) should be scaled when transport properties are scaled.

Our methodology relates gas-saturation maps to our CRM patchy saturation model through the autocorrelation function. This method allows us to estimate the amount of phase-velocity dispersion and attenuation because of the mesoscopic flow associated with frictional loss for realistic fluid distributions. These estimates are consistent with our current understanding of wave attenuation in patchy saturated porous rocks (Toms et al., 2006, 2007). Only a small percentage of gas distributed in the pore fluid is needed to produce significant attenuation (for the rock under investigation in the ultrasonic frequency band; Figure 11). Conversely, larger amounts of gas reduce the effect of wave-induced flow (gas saturation on the order of $35 \%$; Figure 13).

In future work, we plan to make acoustic measurements in addition to performing saturation experiments and taking tomographic images. However, at ultrasonic frequencies, attenuation mechanisms of a different nature such as attenuation from squirt flow (Gist, 1994) or scattering (Gelinsky et al., 1998) can occur. By comparing experimentally derived velocity and attenuation against theoretical estimates produced using CRM (having incorporated spatial information extracted from tomographic images), we may gain insight into whether mesoscopic fluid flow is a controlling factor on the acoustic properties of partially saturated rock. Large discrepancies between our theoretical estimates and experimental observation may indicate that mesoscopic fluid flow does not dominate or, perhaps, that frame heterogeneities need to be accounted for. The advantage of using CRM for this analysis is that, unlike other patchysaturation models (e.g., White et al., 1976), we have defined the fluid distribution experimentally; thus, no parameters need to be tampered with to match theoretical and experimental measurements.

\section{CONCLUSIONS}

We have used fluid-distribution maps obtained from X-ray tomography imaging to infer relevant statistical measures that describe fluid-saturation patterns. In a second step, we used these statistical measures to model the acoustic response of the partially saturated rock. Our analysis demonstrates that the autocorrelation function can be approximated well by a single Debye correlation function or superposition of two such functions. We evaluated the 
characteristic length scales and showed their sensitivity (and significance) with respect to the percentage of gas saturation. We observed an almost linear decrease of the Debye correlation length with increasing gas saturation.

We concluded that correlation function and correlation length identified from gas-saturation maps provide useful statistical information to quantify fluid-saturation patterns and changes in these patterns at the mesoscale. Hence, models based on the theory of statistical wave propagation have the potential to simulate the acoustic signatures of partially saturated rocks accurately. From the limestone sample data analyzed, we conclude that mesoscopic heterogeneity (fluid patches) on the millimeter scale can produce significant wave attenuation at sonic frequencies.

\section{ACKNOWLEDGMENTS}

The work of J.T.S was performed while receiving a CSIRO studentship and WACUPS postgraduate scholarships. This study was supported by the Curtin Reservoir Geophysics Consortium and by the Deutsche Forschungsgemeinschaft (contract MU1725/1-3). We thank the associate editor and anonymous reviewers for their comments and suggestions, which improved our manuscript.

\section{REFERENCES}

Arns, C. H., F. Bauget, A. Limaye, A. Sakellariou, T. J. Senden, A. P. Sheppard, R. M. Sok, W. V. Pinczewski, S. Bakke, L. I. Berge, P. E. Oren, and M. A. Knackstedt, 2005, Porescale characterization of carbonates using Xray microtomography: Society of Petroleum Engineers, 90368-PA.

Arns, C. H., M. A. Knackstedt, W. V. Pinzewski, and E. G. Garboczi, 2002, Computation of linear elastic properties from microtomographic images: Methodology and agreement between theory and experiment: Geophysics, 67, 1396-1405.

Arns, C. H., M. A. Knackstedt, W. V. Pinzewski, and W. B. Lindquist, 2001, Accurate computation of transport properties from microtomographic images: Geophysical Research Letters, 28, 3361-3364.

Arns, C. H., M. A. Knackstedt, W. V. Pinzewski, and N. S. Martys, 2004, Virtual permeatry of microtomographic images: Journal of Petroleum Science and Engineering, 45, 41-46.

Berryman, J., and J. Blair, 1986, Use of digital image analysis to estimate fluid permeability of porous materials: Application of two point correlation functions: Journal of Applied Physics, 60, 1930-1938.

Brie, A. F., A. F. Pampuri, F. Marsala, and O. Meazza, 1995, Shear sonic interpretation in gas bearing sands: Society of Petroleum Engineers, 30595-MS

Bruhn Building Stone, 2008, Contact Us, http://www.bruhn.com.au/contact.html, accessed 18 November 2008.

Cadoret, T., D. Marion, and B. Zinszner, 1995, Influence of frequency and fluid distribution on elastic wave velocities in partially saturated limestones: Journal of Geophysical Research, 100, 9789-9803.

Cadoret, T., G. Mavko, and B. Zinszner, 1998, Fluid distribution effect on sonic attenuation in partially saturated limestones: Geophysics, 63, $154-160$.

Debye, P., and A. M. Bueche, 1949, Scattering by an inhomogeneous solid: Journal of Applied Physics, 20, 518-525.

De Gryze, S., L. Jassogne, J. Six, H. Bossuyt, M. Wevers, and R. Merckx, 2006, Pore structure changes during decomposition of fresh residue: X-ray tomography analyses: Geoderma, 134, 82-96.

Dunsmuir, J. H., S. R. Ferguson, K. M. D'Amico, and J. P. Stokes, 1991, $\mathrm{X}$-ray microtomography:A new tool for the characterization of porous media: Society of Petroleum Engineers, 22860-MS.

Ehrenberg, S. N., 2007, Whole core versus plugs: Scale dependence of porosity and permeability measurements in platform carbonates: AAPG Bulletin, 91, 835-846.

Gassmann, F., 1951, Elastic waves through a packing of spheres: Geophysics, 16, 673-85.

Gelinsky, S. S., A. Shapiro, T. M. Müller, and B. Gurevich, 1998, Dynamic poroelasticity of thinly layered structures: International Journal of Solids and Structures, 35, 4739-4752.

Gist, G. A., 1994, Interpreting laboratory velocity measurements in partially gas-saturated rocks: Geophysics, 59, 1100-1109.

Homsy, G. M., 1987, Viscous fingering in porous media: Annual Review of Fluid Mechanics, 19, 271-311.

Hounsfield, G. N., 1972, A method of and apparatus for examination of a body by radiation such as X- or gamma radiation: U.K. Patent 1283915.

Johns, R. A., J. S. Steude, L. M. Castanier, and P. V. Roberts, 1993, Nondestructive measurements of fracture aperture in crystal-line rock cores using X-ray computed tomography: Journal of Geophysical Research, 98, 1889-1900.

Johnson, D. L., 2001, Theory of frequency dependent acoustics in patchysaturated porous media: Journal of the Acoustical Society America, 110, 682-694.

Ketcham, R. A., and W. D. Carlson, 2001, Acquisition, optimization and interpretation of X-ray computed tomographic imagery: Applications to the geosciences: Computers and Geosciences, 27, 381-400.

Mavko, G., and T. Mukerji, 1998, Bounds on low frequency seismic velocities in partially saturated rocks: Geophysics, 63, 918-924.

Monsen, K., and S. E. Johnstad, 2005, Improved understanding of velocitysaturation relationships using 4D computer-tomography acoustic measurements: Geophysical Prospecting, 53, 173-181.

Müller, T. M., and B. Gurevich, 2004, One-dimensional random patchy saturation model for velocity and attenuation in porous rocks: Geophysics, 69 , 1166-1172.

- 2005, Wave-induced fluid flow in random porous media: Attenuation and dispersion of elastic waves: Journal of the Acoustical Society of America, 117, 2732-2741.

Müller, T. M., G. Lambert, and B. Gurevich, 2007, Dynamic permeability of porous rocks and its seismic signatures: Geophysics, 72, no. 5, E149E158.

Neuman, S. P., 1994, Generalised scaling of permeabilities: Validation and effect of support scale: Geophysical Research Letters, 21, 349-352.

Norris, A. N., 1993, Low-frequency dispersion and attenuation in partially saturated rocks: Journal of the Acoustical Society of America, 94 359-370.

Oh, W., and W. B. Lindquist, 1999, Image thresholding by indicator kriging: IEEE Transactions on Pattern Analysis and Machine Intelligence, 21, 590-602.

Olafuyi, A. O., A. P. Sheppard, C. H. Arns, R. M. Sok, Y. Cinar, M. A. Knackstedt, and W. V. Pinczewski, 2006, Experimental investigation of drainage capillary pressure using digitized tomographic images: Society of Petroleum Engineers, 99897-MS.

Orsi, T. H., and A. Anderson, 1999, Bulk density calibration for X-ray tomographic analyses of marine sediments: Geo-Marine Letters, 19, 270-274.

Peyton, R. L., B. A. Haeffner, S. H. Anderson, and C. J. Gantzer, 1992, Applying X-ray CT to measure macropore diameters in undisturbed soil cores: Geoderma, 53, 329-340.

Sengupta, M., and G. Mavko, 2003, Impact of flow-simulation parameters on saturation scales and seismic velocity: Geophysics, 68, 1267-1280.

Shepp, L. A., and B. F. Logan, 1974, Reconstructing interior head tissue from $\mathrm{X}$-ray transmissions: IEEE Transactions on Nuclear Science, NS-21, 228 236.

Toms, J., T. M. Müller, R. Ciz, and B. Gurevich, 2006, Comparative review of theoretical models for elastic wave attenuation and dispersion in partially saturated rocks: Soil Dynamics and Earthquake Engineering, 26, 548-565.

Toms, J., T. M. Müller, and B. Gurevich, 2007, Seismic attenuation in porous rocks with random patchy saturation: Geophysical Prospecting, 55, 671-678.

Torquato, S., 2002, Random heterogeneous materials: Microstructure and macroscopic properties: Springer-Verlag New York.

Turner, M. L., L. Knüfing, C. H. Arns, A. Sakellariou, T. J. Senden, A. P. Sheppard, R. M. Sok, A. Limaye, W. V. Pinczewski, and M. A. Knackstedt, 2004, Three dimensional imaging of multiphase flow in porous media: Physica A, 339, 166-172.

Wellington, S. L., and H. J. Vinegar, 1987, X-ray computerized tomography: Journal of Petroleum Technology, 39, 885-898.

White, J. E., N. G. Mikhaylova, and F. M. Lyakhovitskiy, 1976, Low-frequency seismic waves in fluid-saturated layered rocks: Physics of the Solid Earth Transactions, 11, 654-659.

Withjack, E. M., C. Devier, and G. Micheal, 2003, The role of X-ray computed tomography in core analysis: Society of Petroleum Engineers, 83467.

Wood, A. B., 1941, A textbook of sound: Bell. 